\title{
Major Phonetic Processes in Sabzevari Dialect
}

\author{
Anita Aldaghi \\ Payame Noor University, Sabzevar, Iran \\ Email: aldaghipf@yahoo.com \\ Nastaran Tavakoli \\ Azad Islamic University, Fars Branch for Science \&Research, Iran \\ Email: nastaran_ty@yahoo.com
}

\begin{abstract}
Sabzevar with an area of 19500 square kilometer is located in Northeast Iran, In Khorasan Razavi province. Northward, it is bounded to Quchan and Esfarayen, Eastward to Neyshabour, Southward to Kashmar and Shahrood and Westward to Semnan province. With a phonetic approach and after giving a short definition for "dialect", this piece of writing attempts to introduce the most prominent phonetic processes of Sabzevari dialect -one of the Western new Iranian dialects- and introduce vocal changes of this dialect compared with Standard Persian. The findings of this research show that in Sabzevari dialect "mutation" is the main phonetic process and "metathesis" is of the lowest frequency.
\end{abstract}

Index Terms - Iranian dialects, Sabzevari dialect, phonetic processes, vocal change

\section{INTRODUCTION}

Sabzevar with an area of 19500 square Kilometer is located in Northeast Iran, in Khorasan Razavi province. Northward, It is bounded to Quchan and Esfarayen, Eastward to Neyshabour, Southward to Kashmar and to Shahrood and Westward to Semnan province.

Sabzevar city is located on Mashhad- Shahrood way with $950 \mathrm{~m}$ above sea level. It is $226 \mathrm{~km}$ from Sabzevar to Mashhad and $664 \mathrm{~km}$ to Tehran. It is bound to Joghatay Mountains on the North and to Koohmish on the south. There are fertile plains and prairies within the high lands. The most important seasonal river is Kalshoor originated from Binalood Mountains in North of Neyshaboor and flowing to the West. After Mongols invasion to Iran, Sabzevar was called Sarbedaran for a while to the memorial of its brave defendants called "Sarbedaran". It was also called" Sasoyeabad, Biyeh and Beihagh.

Most people of Sabzevar and the surrounding villages talk in Sabzevari dialect which is a variety of modern western Iranian dialects. But in Northern villages different varieties of Turkish as well as Kurmanji are also prevalent. As other Iranian dialects and accents, Sabzevari dialect is moving backward affected by Standard Persian and only businessmen, craftsmen, and representatives of Sabzevar former generations talk it.

Since "The major part of history and cultural identity of people is hidden in their language and dialect"(Cristal, 2006, P. 7) and as Oliver Vandel Holmes puts it, "Each dialect is a temple containing the soul of its speakers and guards them."(Cristal, same, P. 85) Dialectology, as a branch of applied linguistics holds a high position in modern linguistics. "Studying dialects and their various aspects not only provides raw materials for other language researches, but also can help comprehend other language universals. Therefo re the study of major phonetic processes in Sabzevari dialect and indicating their similarities and differences with standard Persian are the main subject of this article.

It should be noted that the language data used in this study are mainly gathered through field study, that is recording illiterate or little-literate local native speakers talks and interviewing in addition to getting benefit of the linguistic perception of one of the writers who is the native speaker of Sabzevari dialect to have an even more precise analysis of the data.

\section{DIALECT}

Dialect is sometimes defined as an incorrect and inferior variety of standard language common in remote regions. While in linguistic point of view, dialects are to be considered as language, even though they are not "Formal" languages.

Dialects of a language are, in fact, varieties of that language with differences in phonetics, structure and vocabulary. If the difference among two or more variety is limited only to phonetics, they are called accents. Each language can have different dialects and accents and at the same time each dialect can have a variety of accents.

Dialects are divided in two groups: Social and Local. Social dialects are those current among people with common social, economic or cultural positions. Local dialects are those common in different geographical regions. (Fathi, 2001, P. 1) 


\section{LITERATURE}

"The history of systematized studies of dialects goes back to more than one century." (Mohebbi Bahmani, 2005, P. 21) a comprehensive list of the researches made on Iranian dialects are mentioned in "A list of Iranian languages and Accents" by Ross (1953) in the first chapter of the first volume of Iranzamin Glossary and" Iranian languages and accents bibliology : by Afshar (1955) in the first chapter of third volume of Iranzamin Glossary.

Among the researches made on Sabzevari dialect so far, we can refer to:

Mr. Behroz Ashnay Ghasemi Thesis for receiving Postgraduate Degree (1987) titled "Morphological Study of Sabzevari Dialect in First Volume of Kelidar" (Books 1\&2)

Parvin Sedayee Postgraduate thesis (1987) titled "Morphological Study of Sabzevari Dialect in First Volume of Kelidar " (Books 3\&4)

Zahra Safavi Mobarahan (1987) Postgraduate thesis titled: "Sabzevari Dialect in Kelidar" (Books 5\&6)

Yasaman Taghibeigi Postgraduate thesis (1987) titled "Morphological Study of Sabzevari Dialect in Kelidar" (Books 7\&8) and Alireza Sahebinezhad Postgraduate thesis (1994) titled "Study of Sabzevari Dialect"

Azam Staji has studied Vocal Coordination in Sabzevari Dialect in the article with the same title. Other researches made in this regard are: "Linguistic Study of Sabzevar Dialect "(2002) by Abolfazl Broghani and "Sabzevar Local Glossary" including Sabzevari Dialect Vocabulary and Expressions with samples of local poem (1996) and "Analytical Glossary of Sabzevari Proverbs" (1999) written by Hassan Mohtasham.

\section{SABZEVARI Dialect SOUND SySTEM}

\section{A. Consonants}

As in Persian, this dialect contains 23 Consonants as follows.

\begin{tabular}{|c|c|c|}
\hline $\begin{array}{l}\text { Phone Persian } \\
\text { Equivalent }\end{array}$ & Phonetic description & Phonetic Sign \\
\hline ب & Voiceless, Bilabial, Stop & $/ \mathrm{p} /-$ \\
\hline ب ب & Voiced, Bilabial, Stop & $\mathrm{b} /-$ \\
\hline ت، ط & Voiceless, Dental, Stop & $/ \mathrm{t} / \mathrm{-}$ \\
\hline 2 & Voiced, Dental, Stop & $/ \mathrm{d} /-$ \\
\hline 5 & Voiceless, Velar,Stop & $/ \mathrm{k} /-$ \\
\hline 3 & Voiced, Velar,Stop & $/ \mathrm{g} /-$ \\
\hline ق، غ & Voiced, Uvular ,Stop & /q/- \\
\hline 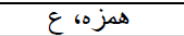 & Voiceless, Glottal,Stop & $1 \%$ \\
\hline ह & Voiceless ‘Affricate, Alveopalatal,Stop & $/ \overline{\mathrm{c}} /-$ \\
\hline ج & Voiced‘Affricate Alveopalatal ,Stop & ij - \\
\hline فت & Voiceless ،Labiodentals ،Fricative & $\mathrm{ff}-$ \\
\hline و & Voiced ‘Labiodentals ‘Fricative & $/ \mathrm{V} /-$ \\
\hline 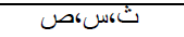 & Voiceless 'Alveolar'Fricative & $/ \mathrm{s} /-$ \\
\hline 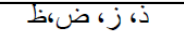 & Voiced 'Alveolar'Fricative & $\mid \mathrm{z} /-$ \\
\hline شَ & Voiceless ‘Fricative Alveopalatal & $/ 5 /-$ \\
\hline$\dot{j}$ & Voiced ' Alveopalatal ' Fricative & $\mid \check{z}-$ \\
\hline$\dot{\tau}$ & Voiceless ‘Uvular, ‘ Fricative & $/ \mathrm{x} /-$ \\
\hline 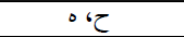 & Voiceless ، Glottal ، Fricative & $\mathrm{h} / \mathrm{-}$ \\
\hline 5 & Voiced ‘Bilabial ‘Nasal & $/ \mathrm{m} /-$ \\
\hline ن & Voiced ‘Dental ‘ Nasal & $/ \mathrm{n} /-$ \\
\hline J & Voiced 'Alveolar ‘Lateral & 11- \\
\hline $\mathrm{J}$ & Voiced · Alveolar ‘ Trill & $\mid \mathrm{r} / \mathrm{-}$ \\
\hline ज & $\begin{array}{l}\text { ‘Palatal'(Semi-Vowel) Approximant } \\
\text { Voiced }\end{array}$ & $/ y /-$ \\
\hline
\end{tabular}

It should be noted that:

a) Some words in this dialect are pronounced with more force like /qand/ in which phoneme q is pronounced with more force and is an allophone of /q/ and makes no meaning distinction.

b) In some words (like /haykal/, phoneme /h/ is pronounced as in Arabic language which makes no meaningful distinction and therefore is considered an allophone.

Points on Consonant Distribution:

All existing Consonant in this dialect can appear at the beginning, in the middle and at the end of the word like their counterparts in Standard Persian.

Phoneme/'/can be omitted in the middle and at the end of the word, as: /do'a/:/do'ā /:Pray, /šam'/ Candle /ša:m/:

In Sabzevari dialect $/ \mathrm{h} /$ in the middle of the word is usually omitted as: /behtar/:/be:tar/ "Better"

This phoneme is also omitted at the end of the word after phoneme/ā / like

Well /čā/ Road and،/rā/

B. Vowels 
Like standard Persian, this dialect has 6 simple vowel (Short and long) as follows.

\begin{tabular}{|c|c|c|}
\hline \multicolumn{3}{|c|}{ SIMPLE VOWELS: } \\
\hline $\begin{array}{c}\text { Phone Persian } \\
\text { equivalent }\end{array}$ & Phonetic Description & Phonetic Sign \\
\hline إى & Closed, Front, Spread & /i/ g/i/ - \\
\hline I & Semi-c losed ,Front, spread & $/ \mathrm{e} /-$ \\
\hline$T$ & Open , Front , spread & $/ \mathrm{a} /-$ \\
\hline$T$ & Open, back, spread & $/ \overline{\mathrm{a}} /-$ \\
\hline$T$ & Semi-closed, back, Round & $/ 0 /-$ \\
\hline او & Closed, Back, Round & $/ \overline{\mathrm{u}} / \mathrm{g} / \mathrm{u} / \mathrm{-}$ \\
\hline
\end{tabular}

\begin{tabular}{|c|c|c|c|}
\hline Meaning & Example & $\begin{array}{c}\text { Phone Persian } \\
\text { equivalent }\end{array}$ & Phonetic Sign \\
\hline Wine & may/ & जा & /ay/- \\
\hline Link & /peyvan/ & إى & /ey/ - \\
\hline Second & /doyyom/ & اा & /oy/ - \\
\hline Slipper & /dempāyil & ایى & /āy/ - \\
\hline $\begin{array}{c}\text { Morbouy( A desert } \\
\text { plant with yellow } \\
\text { flowers) }\end{array}$ & /murbuy/ & اوى & /uy / - \\
\hline Fever & /tow/ & أو & /ow/ - \\
\hline Shoe & /kawš/ & أو & /aw/- \\
\hline Father & /piyar/ & اي & /iy/ - \\
\hline
\end{tabular}

As in standard Persian, no word begins with vowel in this dialect.

\section{MAJOr PhONETIC Processes in SABZEVARi Dialect}

"Phonetic units affected by Syntagmatics are changed. These changes are called Phonetic processes." (Haghshenas, 1992, P. 147)

\section{A. Elision}

Sometimes under certain conditions a phone is omitted from speech chain. Generally there are two elision processes in language.

1. Historical Elision: In which a phone is omitted from certain phonetic chains during the time, having passed historical procedures of the phone subject to certain historical-linguistic structures. (same, P. 157)

2. Structural elision: which is pursuant to the language sound system and governs phone combination in speech chain, that is, whenever an syntagmatics between segments occur, that is contrary to the language sound system or seems difficult based on language phonetic nature, a phonetic unit is omitted from speech chain to solve the problem.(Same, P. 158) beside historical and structural elision, there also exist some exceptional elisions for which no justification can be found. (Same,P. 159)

a) Middle Elision:

\begin{tabular}{|c|c|c|}
\hline \multicolumn{3}{|c|}{ CONSONANT ELISION } \\
\hline Meaning & Standard Persian & Sabzevari \\
\hline Akbar, The craftsman & /‘Ostād akbar/ & /"este akbar/ \\
\hline Old & kohne/ & /kona/ \\
\hline \multicolumn{3}{|c|}{ VOICED ELISION: } \\
\hline Meaning & Standard Persian & Sabzevari \\
\hline You, Yourself & /xod-e- šomā/ & /xad šomā/ \\
\hline Scrambled eggs & /xāgine/ & /xāgna/ \\
\hline \multicolumn{3}{|c|}{ VOWEL \&CONSONANT ELISION: } \\
\hline Meaning & Standard Persian & Sabzevari \\
\hline Veil & /čādor/ & /čār// \\
\hline Mohammad & /mohammad/ & $/ \mathrm{mammad} /$ \\
\hline
\end{tabular}

b). Ending Elision

\begin{tabular}{|c|c|c|}
\multicolumn{5}{|c|}{ VoICED ELISION: } \\
\hline Meaning & Standard Persian & Sabzevari \\
\hline Gluttonous & /xorande/ & /xorand/ \\
\hline
\end{tabular}




\begin{tabular}{|c|c|c|}
\hline & ( & \\
\hline Meaning & Standard Persian & Sabzevari \\
\hline Miserable & /badbaxt/ & /bedbax/ \\
\hline Kissed & /busid/ & /busi/ \\
\hline Cooked & poxt/ & pox \\
\hline Link & /peyvand/ & /peyvan/ \\
\hline Soul & jān/ & $/ \mathrm{jo} /$ \\
\hline Wood & /čub/ & $/ \check{c} \mathrm{u} /$ \\
\hline Street & /xiyābān/ & /xiyābo/ \\
\hline said & /goft/ & /gof/ \\
\hline Bread & /nān/ & /no/ \\
\hline Deserted & /virān/ & $/$ vevrū/ \\
\hline
\end{tabular}

\section{B. Addition}

Sometimes in certain conditions a phone (or a segment) is added to the speech chain. This process is called "Addition". Like elision, addition follows language sound systems. That is whenever a syntagmatics occurs between language segments which is regarded as heavy based on the language phonetic nature or is contrary to language sound system, a chain segment is added to speech chain to remove this problem.(Same)

1. Beginning Addition:

Sometimes an inflectional" b" is added to the beginning of past tense verbs.

\begin{tabular}{|c|c|c|}
\hline Meaning & Standard Persian & Sabzevari \\
\hline I read & xāndam/ & /boxondum/ \\
\hline I went & /raftam/ & /beraftum/ \\
\hline I escaped & gorīxtam/ & /bogruxtum/ \\
\hline
\end{tabular}

2. Middle Addition:

VOWELS:

\begin{tabular}{|c|c|c|}
\hline Meaning & Standard Persian & Sabzevari \\
\hline wide & /pahn/ & / čarob/ \\
\hline Fat & /čarb/ & /zoher/ \\
\hline Noon & /zohr/ & /qaher/ \\
\hline On odd & /qahr/ & vazen/ \\
\hline Weight & /vazn/ & \\
\hline
\end{tabular}

CONSONANT:

\begin{tabular}{|c|c|c|}
\hline Meaning & Standard Persian & Sabzevari \\
\hline Picking & /čidan/ & čindan/ \\
\hline Abscess & /domal & $/$ dembal \\
\hline All & /hame/ & hama/ \\
\hline
\end{tabular}

3. Ending Addition

Consonant:

\begin{tabular}{|c|c|c|}
\hline Meaning & Standard Persian & Sabzevari \\
\hline Jinn & /jen/ & /̌̌end/ \\
\hline Lawn & /čamand/ \\
\hline What do you like? & če-mixāhi/ & češ-mexi/ \\
\hline Living room & /nešiman/ & /ničimand \\
\hline
\end{tabular}

\section{Vowel Harmony}

In vowel harmony a vowel affected by another vowel in adjacent syllable loses some of its phonetic features or takes the features of the adjacent vowel or takes features similar to that. Vowel harmony can be progressive or regressive. (Haghshenas, 1992, P. 155,156)

SOME EXAMPLES OF PROGRESSIVE VOWEL HARMONY:
\begin{tabular}{|c|c|c|}
\hline Meaning & Standard Persian & Sabzevari \\
\hline Why & $/$ čerā/ & $/$ čere/ \\
\hline Grate & rande/ $/$ randa/ \\
\hline Book-seller & ketāb foruš/ & kotāb foroš/ $/$ \\
\hline I said & $/$ goftam/ & $/$ goftom/ \\
\hline
\end{tabular}


SOME EXAMPLES OF REGRESSIVE VOWEL HARMONY:

\begin{tabular}{|c|c|c|}
\hline Meaning & Standard Persian & Sabzevari \\
\hline Fire & T'ātašs/ & T'atašs \\
\hline Came & /'āmad/ & /'ama/ \\
\hline Iron & T'āhan & T'ahan \\
\hline Founta in-Pen & /xodnevis/ & /xādnivis/ \\
\hline
\end{tabular}

\section{Assimilation}

Sometimes a consonant loses some of its phonetic features in syntagmatic with another consonant and takes the phonetic features of its adjacent consonant instead. This process, perhaps one of phonetic universals, is called" Assimilation". If this process causes one of consonants to turn completely into another consonant, it is called "Complete Assimilation". However, if the assimilation does not end in complete assimilation, then it is called "Partial Assimilation." (Same, P. 152)

Assimilation can be progressive or regressive. In progressive assimilation of two syntagmatic consonants, the one in the first place remains fixed and unchanged and the one in the second place is assimilated. In regressive assimilation of two syntagmatic consonants, the one in the second place remains fixed and unchanged and the one in the first place is assimilated.

INSTANCES OF COMPLETE ASSIMILATION:

\begin{tabular}{|c|c|c|}
\hline Meaning & Standard Persian & Sabzevari \\
\hline Worse & /badtar/ & /battar/ $/$ bezzāt/ \\
\hline Ill-natured & /badzāt/ & /bajjens/ \\
\hline Wicked & /badjens/ $/$ bajjūr/ \\
\hline Wrong & /badjūr/ & . \\
\hline
\end{tabular}

INSTANCES OF INCOMPLETE ASSIMILATION:
\begin{tabular}{|c|c|c|}
\hline Meaning & Standard Persian & Sabzevari \\
\hline Pants & /tonbān/ & / $/$ dombo/ $/$ \\
\hline Trace & /donbāl & /qembar/ \\
\hline Ghanbar & /qanbar/ & \\
\hline
\end{tabular}

\section{E. Consonant-vowel Assimilation}

It is possible that regressive and progressive assimilation occur between a syntagmatic consonant and vowel. This frequently happens in Persian. For instance, in syntagmatics with all palatal consonants/s/ ,/z/ ,/c/ and /j/ ,/e/ loses its half-closed feature and turns into "closed" although naturally they are pronounced closer than /e/ because they are consonants. (Same, P. 154)

\begin{tabular}{|c|c|c|}
\hline Meaning & Standard Persian & Sabzevari \\
\hline Eye & /čašm/ & /čš/ \\
\hline Boots & /čakme/ & /čikma/ \\
\hline Gloves & /dastkeš/ & /diskaš/ \\
\hline
\end{tabular}

\section{F. Consonants Dissimilation}

In this process which is contrary to assimilation, a consonant, common with its syntagmatic consonant in one or some phonetic features loses some of its common features and gain other phonetic features. Dissimilation process can be progressive or regressive.(Same, P. 155). This process is much more unusual than assimilation.(Arlato, 1994, P.125)

\begin{tabular}{|c|c|c|}
\hline Meaning & Standard Persian & Sabzevari \\
\hline Map & /naq̌̌e/ & /nexša/ \\
\hline Joy & /vajd/ & važd/ \\
\hline Conscience & /vojdān/ $/$ vān/ \\
\hline Time & /vaqt/ & vax/ \\
\hline
\end{tabular}

In all said above examples the assimilation is in regressive type.

\section{G. Mutation}

"Sometimes in speech chain a chain segment turns into another segment with no justification within assimilation, dissimilation, vowel-consonant harmony or other processes. (Haghshenas, 1992, P. 160) This process is called "Mutation." The only justification for this process can be the inadequacy of phonetic chains.

1). Vowel Mutation: 
$1-/ \mathrm{A} / \rightarrow / \mathrm{E} /$

\begin{tabular}{|c|c|c|}
\hline Meaning & Standard Persian & Sabzevari \\
\hline Morals & /'axlāq/ & /'exlāq/ \\
\hline If & /'agar/ & /'eger/ \\
\hline Ax & /tabar/ & / tevar/ \\
\hline Pants & /šalvār/ & /mār/ \\
\hline Unless & /magar/ & /mege/
\end{tabular}

$2-/ \mathrm{A} / \rightarrow / \mathrm{O} /$

\begin{tabular}{|c|c|c|}
\hline Meaning & Standard Persian & Sabzevari \\
\hline Spring & /bahār/ & /bohār/ \\
\hline Pray & /namāz/ & /nomāz/ \\
\hline
\end{tabular}

$3-/ \mathrm{E} / \rightarrow / \mathrm{I} /$

\begin{tabular}{|c|c|c|}
\hline Meaning & Standard Persian & Sabzevari \\
\hline Razor & /tr $/ /$ te $\gamma /$ \\
\hline Scream & /ji $/$ & $/ \mathrm{je} /$ \\
\hline
\end{tabular}

$4-/ \mathrm{E} / \rightarrow / \mathrm{A} /$

\begin{tabular}{|c|c|c|}
\hline Meaning & Standard Persian & Sabzevari \\
\hline A saw & /'arre/ & /'arra/ \\
\hline Cream & /xāme/ & /xāma/ \\
\hline Letter & /nāme/ & /nāma/ \\
\hline
\end{tabular}

5 - $/ \mathrm{E} / \rightarrow / \mathrm{O} /$

\begin{tabular}{|c|c|c|}
\hline Meaning & Standard Persian & Sabzevari \\
\hline Book & $/$ ketāb/ & $/$ kotāb/ \\
\hline Tall & $/$ derāz/ & $/$ dorāz/ \\
\hline
\end{tabular}

$6-/ \mathrm{O} / \rightarrow / \overline{\mathrm{A}} /$

\begin{tabular}{|c|c|c|}
\hline Meaning & Standard Persian & Sabzevari \\
\hline Fountain Pen & /xodnevis/ & xādnivis/ \\
\hline
\end{tabular}

$7-/ \bar{A} / \rightarrow / E /$

\begin{tabular}{|c|c|c|}
\hline Meaning & Standard Persian & Sabzevari \\
\hline Ewer & /'āftābe/ & /'eftave/ \\
\hline Game & /bāzi/ & /bezi \\
\hline Sweep & /jāru/ & /jeru/ \\
\hline Carpet & /qā̄i/ & /qeli/ \\
\hline
\end{tabular}

$8-/ \bar{a} / \rightarrow / 0 /$

\begin{tabular}{|c|c|c|}
\hline Meaning & Standard Persian & Sabzevari \\
\hline Five Hundred & pānsad & ponsad/ \\
\hline Duck & /morqābi/ & /morqovi/ \\
\hline
\end{tabular}

9- $/ \overline{\mathrm{A}} / \rightarrow / \mathrm{A} /$

\begin{tabular}{|c|c|c|}
\hline Meaning & Standard Persian & Sabzevari \\
\hline Home & /xāne/ & xana/ \\
\hline Grain & /dāne/ & /ana/ \\
\hline Womanly & zanāne/ & /zanana/ \\
\hline Comb & ne/ā̌s/ & šana/ \\
\hline
\end{tabular}

10-/U/ $\rightarrow$ /E/

\begin{tabular}{|c|c|c|}
\hline Meaning & Standard Persian & Sabzevari \\
\hline Pottage & $/$ šurbā/ & $/$ šervā/ $/$ \\
\hline
\end{tabular}

$11-/ \mathrm{U} / \rightarrow / \mathrm{O} /$

\begin{tabular}{|c|c|c|}
\hline Meaning & Standard Persian & Sabzevari \\
\hline Teapot & /quri & /qori $/$ \\
\hline Book Seller & ketāb foruš/ & /kotāb foroš/ \\
\hline
\end{tabular}

$12-/ \mathrm{U} / \rightarrow / \mathrm{I} /$

\begin{tabular}{|c|c|c|}
\hline Meaning & Standard Persian & Sabzevari \\
\hline Pennyroyal & /pune/ & pina/ \\
\hline Coffin & /tābut/ & /tābid/ \\
\hline Knife & $/$ čāqu/ & čeqi \\
\hline Knee & $/$ zānū/ & /zeni \\
\hline Bride & arus/// $/ /$ & /'aris/ \\
\hline
\end{tabular}


$13-/ \bar{I} / \rightarrow / E /$

\begin{tabular}{|c|c|c|}
\hline Meaning & Standard Persian & Sabzevari \\
\hline Shovel & $/ \mathrm{b} \overline{1} \mathrm{l} /$ & bell \\
\hline Patch & /pālīz/ & /pelez/ \\
\hline Forehead & pīšnāni & pešeni \\
\hline Sharp & $/$ tìz & /tez/ \\
\hline Late & $/ \mathrm{d} \overline{\mathbf{r}} /$ & $/ \mathrm{der} /$ \\
\hline Garlic & $/ \mathrm{sin} /$ & $/ \mathrm{ser} /$ \\
\hline Pin & $/ \mathrm{mix} /$ & $/ \mathrm{mex} /$ \\
\hline That is & /ya'nī/ & /ya:ne/ \\
\hline
\end{tabular}

$14-/ \mathrm{OW} / \rightarrow / \bar{A} /$

\begin{tabular}{|c|c|c|}
\hline Meaning & Standard Persian & Sabzevari \\
\hline Bright & /rowšan/ & /rāšan/ \\
\hline Oil & /row $\gamma$ an $/$ & rā $/$ an $/$ \\
\hline
\end{tabular}

$15-/ \overline{\mathrm{A}} / \rightarrow / \overline{\mathrm{U}} /$

\begin{tabular}{|c|c|c|}
\hline Meaning & Standard Persian & Sabzevari \\
\hline Roof & bām/ & būm/ \\
\hline Cup & $/$ jām/ & $/$ jūm/ \\
\hline Dinner & $/$ šām/ & $/$ šūm/ $/$ \\
\hline Palate & $/$ kām/ & $/$ kūm/ \\
\hline
\end{tabular}

The word "Salam" "Hello" is an exception, it does not change.

$16-/ \mathrm{A} / \rightarrow / \mathrm{U} /$

\begin{tabular}{|c|c|c|}
\hline Meaning & Standard Persian & Sabzevari \\
\hline Has gone & /rafte/ & /rufta/ \\
\hline \multicolumn{3}{|c|}{$17-/ \mathrm{O} / \rightarrow / \mathrm{U} /$} \\
\hline Meaning & Standard Persian & Sabzevari \\
\hline Has said & gofte/ & gufta \\
\hline
\end{tabular}

\section{2) Consonant Mutation}

$1-/ \mathrm{M} / \rightarrow / \mathrm{N} /$

\begin{tabular}{|c|c|c|}
\hline Meaning & Standard Persian & Sabzevari \\
\hline possible & /momken & /monken \\
\hline
\end{tabular}

$2-/ \mathrm{B} / \rightarrow / \mathrm{P} /$

\begin{tabular}{|c|c|c|}
\hline \multicolumn{3}{|c|}{$2-/ \mathrm{B} / \rightarrow / \mathrm{P} /$} \\
\hline Meaning & Standard Persian & Sabzevari \\
\hline Dome & /qobbe/ & /qoppa/ \\
\hline
\end{tabular}

$3-/ \mathrm{B} / \rightarrow / \mathrm{V} /$

\begin{tabular}{|c|c|c|}
\hline \multicolumn{2}{|c|}{$3-/ \mathrm{B} / \rightarrow / \mathrm{v} /$} \\
\hline Meaning & Standard Persian & Sabzevari \\
\hline Open & /bāz/ & $/ \mathrm{vā} /$ \\
\hline Axe & $/$ tabar/ & $/$ tevar/ \\
\hline Pottage & $/$ šrbā/ & $/$ šervā/ $/$ \\
\hline Duck & $/$ morqābi/ & $/$ morqovi \\
\hline
\end{tabular}

$4-/ \mathrm{P} / \rightarrow / \mathrm{F} /$

\begin{tabular}{|c|c|c|}
\hline \multicolumn{3}{|c|}{$4-/ \mathrm{P} / \rightarrow / \mathrm{F} /$} \\
\hline Meaning & Standard Persian & Sabzevari \\
\hline Visible & $/$ peydā $/$ & $/$ feydā $/$ \\
\hline Victory & pirūzi & $/$ firūzi \\
\hline
\end{tabular}

$5-/ \mathrm{T} / \rightarrow / \mathrm{D} /$

\begin{tabular}{|c|c|c|}
\hline Meaning & Standard Persian & Sabzevari \\
\hline Nursing & parastāri & /peresdāri \\
\hline Circumc ision-Party & xatne sūrān/ & /xedne sūri \\
\hline Peach & /šeftāh/ & ofdeli/šs/ \\
\hline Kitchen & /matbax/ & /modbax/ \\
\hline
\end{tabular}

6- /Č/ $\rightarrow / \mathrm{Y} /$

\begin{tabular}{|c|c|c|}
\hline Meaning & Standard Persian & Sabzevari \\
\hline Female animal & $/$ māče/ & $/$ maya/ \\
\hline
\end{tabular}




\begin{tabular}{|c|c|c|}
\hline \multicolumn{3}{|c|}{$7-/ \mathrm{x} / \rightarrow / \mathrm{h} /$} \\
\hline Meaning & Standard Persian & Sabzevari \\
\hline Ditch & /xandaq/ & handaq/ \\
\hline \multicolumn{3}{|c|}{$8-/ \mathrm{d} / \rightarrow / \mathrm{t} /$} \\
\hline Meaning & Standard Persian & Sabzevari \\
\hline Headman & kad-xodā/ & /ket-xodā/ \\
\hline Kick & /lagad/ & /leqat (y)/ \\
\hline Mosque & /masjed/ & /meččet/ \\
\hline \multicolumn{3}{|c|}{$9-/ \mathrm{D} / \rightarrow / \mathrm{Z} /$} \\
\hline Meaning & Standard Persian & Sabzevari \\
\hline Jonbad (Name of a Village & /jonbad/ & /jombaz/ \\
\hline Dome & gonbad/ & gombaz \\
\hline \multicolumn{3}{|c|}{$10-/ \mathrm{D} / \rightarrow / \mathrm{C} /$} \\
\hline Meaning & Standard Persian & Sabzevari \\
\hline Female Donkey & /māde xar/ & meče xar/ \\
\hline \multicolumn{3}{|c|}{$11-/ \mathrm{R} / \rightarrow / \mathrm{L} /$} \\
\hline Meaning & Standard Persian & Sabzevari \\
\hline Fattened Animal & parvār/ & polvār/ \\
\hline Cucumber & /xiyār/ & /xiyāl/ \\
\hline Wall & /divār/ & difāl \\
\hline \multicolumn{3}{|c|}{$12-/ \mathrm{Z} / \rightarrow / \mathrm{J} /$} \\
\hline Meaning & Standard Persian & Sabzevari \\
\hline Ugly & /zešt/ & jǘs \\
\hline \multicolumn{3}{|c|}{$13-/ \mathrm{S} / \rightarrow / \check{\mathrm{S}} /$} \\
\hline Meaning & Standard Persian & Sabzevari \\
\hline Very hard land & /saxt/ & /šax/ \\
\hline \multicolumn{3}{|c|}{$14-/ \check{Z} / \rightarrow / \mathrm{J} /$} \\
\hline Meaning & Standard Persian & Sabzevari \\
\hline Dew & /žāle/ & jala/ \\
\hline Eyelash & /može/ & mija \\
\hline \multicolumn{3}{|c|}{$15-/ \mathrm{K} / \rightarrow / \mathrm{G} /$} \\
\hline Meaning & Standard Persian & Sabzevari \\
\hline Akbar & T'akbar/ & T'egbar/ \\
\hline Bank & /bānk/ & /bāng/ \\
\hline \multicolumn{3}{|c|}{$16-/ \mathrm{G} / \rightarrow / \mathrm{K} /$} \\
\hline Meaning & Standard Persian & Sabzevari \\
\hline Loose & /gošād/ & /kešād/ \\
\hline \multicolumn{3}{|c|}{$17-/ \mathrm{V} / \rightarrow / \mathrm{F} /$} \\
\hline Meaning & Standard Persian & Sabzevari \\
\hline Wall & /divār/ & /difāl/ \\
\hline \multicolumn{3}{|c|}{$18-/ \mathrm{V} / \rightarrow / \mathrm{Y} /$} \\
\hline Meaning & Standard Persian & Sabzevari \\
\hline Second & /dovvom/ & /doyyom/ \\
\hline Third & /sevvom/ & /seyyom/ \\
\hline \multicolumn{3}{|c|}{$19-/ \mathrm{V} / \rightarrow / \mathrm{B} /$} \\
\hline Meaning & Standard Persian & Sabzevari \\
\hline Opium smoking tube & /vāfur/ & /bāfur/ \\
\hline A barrier against water & /varq & barq \\
\hline \multicolumn{3}{|c|}{$20-/ \mathrm{H} / \rightarrow / \mathrm{B} /$} \\
\hline Meaning & Standard Persian & Sabzevari \\
\hline Toilet & mostarāh/ & mosterāb/ \\
\hline \multicolumn{3}{|c|}{$21-/ \mathrm{H} / \rightarrow / \mathrm{Q} /$} \\
\hline Meaning & Standard Persian & Sabzevari \\
\hline Kidding & /mezāh/ & /mezāq/ \\
\hline
\end{tabular}




\begin{tabular}{|c|c|c|}
\hline \multicolumn{2}{|c|}{$22-/ \mathrm{s} / \rightarrow / \mathrm{z} /$} \\
\hline Meaning & Standard Persian & Sabzevari \\
\hline Asqar & /'asqar/ & /'ezqar/ \\
\hline \multicolumn{3}{|c|}{ 23-/L/ $\rightarrow / \mathrm{R} /$} \\
\hline Meaning & Standard Persian & Sabzevari \\
\hline Mantle & /šenel/ & šener/
\end{tabular}

3). Sound Cluster Mutation to a Vowel

\begin{tabular}{|c|c|c|}
\hline Meaning & Standard Persian & Sabzevari \\
\hline Gardener & /bāqbān/ & /bāqvu/ \\
\hline Shop & /dokān/ & /diko/ \\
\hline Hayrack & /kāhdān/ & kādo/ \\
\hline Nail & /nāxon/ & /hayvu/ \\
\hline Animal & /heyvān/ & \\
\hline
\end{tabular}

\section{H. Metathesis}

Sometimes two consonants in a combination change their place by syntagmatic in a way first consonant takes the place of the second and the second consonant takes the place of the first. This process is called" Metathesis" (Haghshenas, 1972, P. 156) the frequency of this phonetic process which is a rather unusual type of sound change is low in Sabzevari dialect compared with other processes.

\begin{tabular}{|c|c|c|}
\hline Meaning & Standard Persian & Sabzevari \\
\hline Photograph & /'aks/ & /'ask/ \\
\hline Wick & /fetile/ & /qolf/ $/$ \\
\hline Lock & /qofl/ & /qeylu/ \\
\hline Kalyan & /qalyān/ & keft/ \\
\hline Shoulder & /ketf/ & na:lat/ \\
\hline Damn & /la nat/ & /noxsa/ \\
\hline Prescription & /nosxe/ & \\
\hline
\end{tabular}

Metathesis is also occurred in the second example.

\section{Compensatory Lengthening}

A special type of change covering both vowels and consonants is a trend called "Compensatory Lengthening. When a vowel is followed by two consonants and one of consonants is omitted, the previous vowel is lengthened to compensate the omitted phoneme.(Arlato, 1994, P. 127)

\begin{tabular}{|c|c|c|}
\hline Meaning & Standard Persian & Sabzevari \\
\hline Better & /behtar/ & /be:tar/ \\
\hline Affection & /mehr/ & /mer/ \\
\hline Seal & /mohr/ & /mor/ $/$ \\
\hline That is & ya'ni & yane/ \\
\hline
\end{tabular}

\section{CONCLUSION}

The finding results indicate that mutation is the major phonetic process and metathesis holds the lowest frequency in this dialect. It should be said that the mutation seen in this dialect is mostly of consonant type.

\section{REFERENCES}

[1] Arlato, Anthony. (1994). An introduction to Historical Linguistics. Translated by Yahya Moddaresi, 1st edition, Research center on Humanities and Culture.

[2] Afshar, Iraj. (1955). Bibliography of Iranian Languages and Dialects. Iranzamin Glossary, 1st book, 3rd Volume.

[3] Samareh, Yadollah . (1985).Persian language Phonetics .1st edition, Tehran, University press.

[4] Haghshenas, Alimohammad. (1992). Phonetics. 3rd edition, Tehran: Agah Publication.

[5] Ross, Nichola. (1953). Iranian languages \& dialects references Iranzamin Glossary, 1st book, 1st Volume.

[6] Fathi, Azar. (2001). A Guide to Dialects collection Tehran: Persian Academy of Language \& Literature.

[7] Crystal, David. (2006). The Language Revolution. Translated by Shahram Naghshe Tabrizi. Tehran: Qoqnous.

[8] Mohebbi Bahmani, Hassan. (2005). Minabi Dialect Linguistic Description \&study. Tehran: Sabzan.

[9] Meshkotod Dini, Mehdi. (2006). Phonology An Introduction. Mashhad: Ferdowsi University Press. 


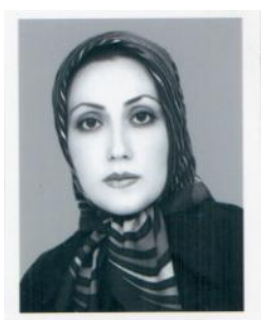

Anita Aldaghi. She was born in September 11, 1975 in one of the Iranian cities called Sabzevar. After secondary school, she went to Azad Islamic University for Continue studies. Undergraduate and postgraduate courses at the University, in Persian Language and Literature field successfully spent. Then went to Tajikistan and her degree Ph.D from the Academy of Sciences of Tajikistan received.

During Education and after, she has compiled twenty-nine different articles in the field of linguistics and Iranian Studies, which has published in scientific centers. For example: Take on building phonetic words and words Equality, in the Kurdish language. Publication four specialized linguistics article in the Journal of the National University of Tajikistan. Publication, two articles, as the article chosen in the Book, comprehensive of Bank Iran Tourism information. Role pronoun question in Persian dialects. Early in 2011, she published a book, with titled culture and myths of ancient's Iran (ajand), she also extensive research in the field of how the ancient Iran burial has done That is editing for publication.

About Resume, these cases can be cited: employee of Payam Noor University Sabzevar from 2003 to now. Teach specialized courses Persian language and literature at the Payam Noor University Sabzevar since 2003. Permanent member of the editorial board of International Journal of gashto gozar.

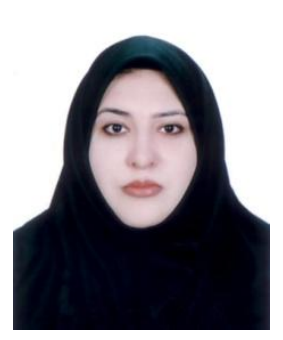

Nastaran Tavakoli. She was born on 07/03/1970 in Shiraz of Iranian city (Fars Province). Bachelor's degree in "Language and Literature of French" from the Department of Literature University of Jundi Shapur Ahwaz (Khuzestan Province) in Iran in 1993 received; Also she received master's degree in culture and ancient languages of Iran "from the School of Literature and Humanities Shiraz University in 2000"; after, Continued education in herself field, and received degree of Ph.D from University of literature Tehran in 2007.

She published 16 articles in the areas of historical linguistics, dialetology. For example: 1) "The Persian Gulf regional dialects and cultural identity; Overview of a building in the ancient dialects of the Persian Gulf region": published in the Proceedings of the Biennial International Conference of Persian Gulf (history, culture and civilization), November 2009: Tehran University.2) "Caucasus; languages Mount (an overview of the role Iranian language in cultural identity of Caucasus), presented as a lecture, "First International Conference on Caucasus Platform", published in the Journal of Research - Institute of Cultural Studies Aran and has won Appreciation, October 2009: Tabriz University.3) (High frequency phonetic processes, in two dialects Kermani Shirazi: one review coping.)Presented as a lecture on "International Conference dialects desert areas of Iran and ready to print in Proceedings of Conference: Persian date Azar 1389: University of Semnan, December 2010.

She has taught as an Assistant professor in the Linguistics Department, Islamic Azad University, Fars Science and Research since 2006. 